J. Sustain. Wireless Syst.

Vol.01/ No. 02

Pages: 116-125

http://irojournals.com/irosws/

DOI: https://doi.org/10.36548/jsws.2019.2.004

\title{
PERFORMANCE EVALUATION OF FOG COMPUTING WITH EDGE DEVICES IN MEDICAL IOT APPLICATION
}

\author{
Dr. G. Ranganathan, \\ Professor, \\ Department of Electronics and Communication Engineering, Gnanamani College of Technology, \\ Namakkal, Tamil Nadu, India - 637018. \\ Email id: profranganathang@gmail.com
}

\begin{abstract}
Monstrous development in the communication and its supporting software's has made our day today necessities which were once in our dream into existence. One such is the internetwork of things. This IoTs which are the a merge of many different technologies is a dais for many tangible commodities that are enabled with embedded computing, information initiated by every such commodities are computed processed and were stored in a cloud in the days past proved to be very successful. But the problem aroused on the clamp down such as latency and heightened bandwidth consumption in which the latency was the very important criteria to be met for the time sensitized information's that were to be processed so there arouse a need to bring down the time interval between the initiation and the response time of information. This becomes more indispensable in sectors like surveillance and medical field. So the paper proposes an intervening computation known as fogging between the cloud and IoT, in order to bring down the latency period in medical field and the performance evaluation are done on the grounds of , latency, bandwidth and energy consumption.
\end{abstract}

Keywords: Internetwork of Things, Fogging, Latency, Bandwidth, Cloud, Medical Field, Energy Consumption..

\section{Introduction}

The acronym IoT which can be unfolded as internet of things is expounded as colossal internet gigantic than the current internet of things. It is the merger of different technologies that are requisite. This internet of things or internetwork of things is envisioned to provide a service at a contemporary level of World Wide Web anatomy. The purview of the computer and their computing devices being connected are enlarged or expanded by the use of internet of things. Supported by both computer, mechanical, digital operations and their capability of transferring information's equip internet of things to elude the man to man and man to computer interactions. It is essentially a broadened dais offered for all the real tangible commodities incorporated with embedded systems, embedded electronics and information technology, so as to make them possess computing basics and consider them as a part of internet of things .these physical commodities that are considered to be the working part of internet of things may distinct in the nature of job the do and might have varied demands on for their job to be done, thus extending their connection which may end up with more loaded working parts in the internet of things. In technical words the internet of things can be expressed as the exclusive way to elevate the various facets of life by systematized use of 
J. Sustain. Wireless Syst.

Vol.01/ No. 02

Pages: 116-125

http://irojournals.com/irosws/

DOI: $\underline{\text { https://doi.org/10.36548/jsws.2019.2.004 }}$

information, network and calculations done with proper set of rules. The internet of things can be contrived in two different methodologies, one is to begin from the initial stages that starting to build the internetwork from the beginning and the next one just to have an expanded purview of the present day internet. The internet of things involve the radio frequency identification device, devices to detect the physical measures of the commodities connected, ways and sources to open out connections between the devices of different standards, dais to accumulate the handled information's after they are being processed by network, communication and data aggregation standards and to provide insightfulness based on the processing done using perceptive methods. The internet of things is a palpable environs of ever increasing entanglement. The next contrive of internet work of things is to have sophisticated devices in our day today lives that is providing with our lives with devices that are instinctive. As told before the merging of different technology will make applications based on the internet of things much facile and swift, which would out turn with an improvisation under various facets of day today life involved activities. The IoTs can be expected to keep in charge of anything, anytime and anyplace connectivity's. The enactments of internet of things may begin from meagre things such as lights, fans to all electronic appliances in a house to evade irregularities in their functioning, in a school by updating proper student information's and school environment to the parents regarding their attendance their monthly marks so on, in business by monitoring and giving necessary adoptions to be done for its easy access and also keeps track of the major disasters to take place. The IoTs involvement is in our everyday essentials that is the everyday travel we take, in every exigency health situations and so on. So they act as a revamp customer service providing an effective usage of technology turning down its misuse. As told in the beginning they are the internet of things is the clubbing of different things or devices with different aspects that work on different tools supported by the knowledge of embedded computing. So they would resemble a large cluster of things so in result there would be generation of lots of data to be processed. The working of these internetwork of things can be segregated into three steps, first is to have the data generated then it is been taken for processing and sent back after process and then the result reached due to processing is given back to the devices, this processing usually takes place under the supervision of cloud computations which look after its processing as well as storage. But due to certain clampdowns of cloud the large data handling and processing and communicating back results with increased time interval between the sensing and generating of information and its response time, large energy consumption and heightened bandwidth consumption so for the efficient handling and have an refined measures of the restraints mentioned above mentioned above the fogging was introduced. Fogging is one of the propitious technologies for the pragmatic worldwide system of internet of things. Its computation are performed in disseminated manner the services such as computation and storage rendered by fogging is always close to the client or near to the user edge rather than sending data far of to be processed. Fog doesn't come as a replacement to the cloud but as an extension of cloud by stretching out cloud computing that is a remote place to its user end.it acts as a transitional layer between the cloud and IoT providing speedy analysis on the data initiated, thus causing the reduction in the to and fro travel time of the data, power and the bandwidth consumed. Here the information initiated are cleaved into three categories as more time sensitized, 
J. Sustain. Wireless Syst.

Vol.01/ No. 02

Pages: 116-125

http://irojournals.com/irosws/

DOI: $\underline{\text { https://doi.org/10.36548/jsws.2019.2.004 }}$

less time sensitized and non-time sensitized. The fog does the proceedings for information's less and more sensitized of time in fog layer and non-sensitized of time is sent to cloud for processing by this the travel time of time sensitized will be reduced and the results are obtained at the user end before the time for the data to be processed elapses or the need for the data to be processed is dead. By this the traffic to the cloud is reduced in turn reducing the bandwidth and the energy consumption is also reduced.

So the paper propose a fogging as an intervening layer between the cloud and the IoT so as to have a reduced latency for the exigent information's that are time sensitized to be handled in medical field .

The paper is ordered as 2. Related works, 3. Proposed, 4.result evaluation and 5.conclusion

\section{Related works}

Mutlag, et.al [1] gives detailed study on the problem issues and challenges on the fog based computing in application involved in health care and offers an effecting assay comparing the cloud computing and fog computing and shows the reduced latency acquired in the fog than the cloud. Elhoseny, et.al [2] discuss on the improved level of security in storage of patient details using encryption and decryption process obtaining an optimal key selection using swarm optimization to demonstrate a betterment in IoT of medical fields. Tran et.al [3] gives the improvement attained in the IoT services supported by multitier fog computation to evolve location, response time and resource consumption of services using resources virtualization. Muthanna, et.al [4] gives insight on how the challenges in internet of things due to the increased number of sensors are solved using computation based on fog under the management of software defining network and does an effective assay on reliability, energy constraints and availability., Wu et.al [5] comes up with the proposal on connection establishment for a standalone local server and internet using internet of things cloud server for web monitoring and mobile applications. Abdellatif, et.al [6] shows the effective usage of implementing weightless classification with accuracy based on edged and data reducing technology that provides with an efficiency in real-time and telemonitoring. Aloi, et.al [7] the inexpensive and validated solution for the medical care applications based on the edge based computation provides a proper flow on the huge amount of data coming from internet of things to internet. Cerina, et.al [8] fog architecture with flexible and multilevel is used in the designing of the smart health applications to overcome the latencies and scaling the capacity of number of devices connected. Nakhkash, et.al [9] gives details on usage of offloading wearable devices to reduce over burden of mobile gateways achieving energy efficiency and compares the gateway energy consumption on multi-threading and single threading execution. Fiandrino, et.al [10] multiple scenario based android application which is supported by fog and mobile cloud computing to overcome the limitations on the use of battery storage and computing also enabled with offloading of wearable device to save the battery energy. Roca, 
J. Sustain. Wireless Syst.

Vol.01/ No. 02

Pages: 116-125

http://irojournals.com/irosws/

DOI: $\underline{\text { https://doi.org/10.36548/jsws.2019.2.004 }}$

et.al [11] gives a new scalable complementation to fog by following new organized policy, virtualization and clustering of the nodes. Newaz, et.al [12] highlights on the expanding network systems on communicating using the integrated passive optical and fog computing method to have a considerable improvement in quality of service. Mahmoud, et.al [13] shows a fog enabled cloud of things offers an energy aware dispersion strategy for internet of things

\section{Proposed Work}

Internetwork of things which constitute an interconnection of devices is purposed for the collection massive consignment of information's to be processed. All these massive load of information has to be processed on the cloud and then stored in it for future reference and the result of processing is to be sent back to the devices. So the cloud that is located in remote location employs a considerable amount of time to execute the task and send back the response. This created untoward mishaps in the user end due to the hold -up on response. The time taken for an information to be processed between cloud and Iot would be given as in equation in (1)

$$
\mathrm{TRT}=\mathrm{St}+\mathrm{Tc}+\mathrm{Pt}+\mathrm{Rc}
$$

Where is the total response time TRT, sensing time St, time taken to travel to cloud Tc, processing time Pt and Rc return time from cloud.

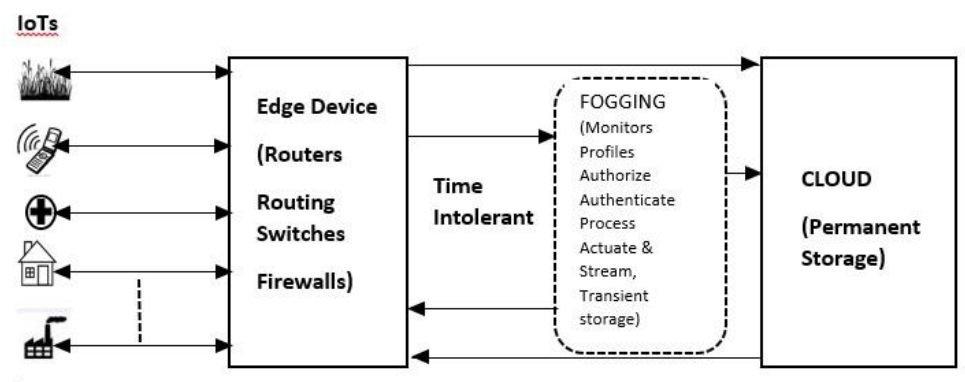

Fig.1 fogging structure

Considering such cases on the exigency medical conditions the results would be worse so to overcome this hold up the paper proposes a fogging as shown in Fig 1 as an intervening layer between the cloud and the IoTs edge devices and elongates the service of the cloud to its user end causing a reduction in the holdup time. It acts as a transient storage device and performs the computation in an accessible distance to the user and sends back the result and encapsulates the particulars of the processed information to the cloud for permanent stor 
J. Sustain. Wireless Syst.

Vol.01/ No. 02

Pages: $116-125$

http://irojournals.com/irosws/

DOI: $\underline{\text { https://doi.org/10.36548/jsws.2019.2.004 }}$

The internetwork of things in medical field offers a better exactness, scope and meticulousness of medical data's by subsume of more well developed and advanced technologies in it. It authorizes the usage of mastery and the priming of a doctor in a better way as to have a better solution to the problems arising, it also enables the swift actions on the utilizations of the information and the devices to have a correct dispersing of the details regarding the patients by bringing the facilities of hospital to the user end providing a better fulfillment and prevention care to its users. The fogging involved in it further provides the in time response for all the exigency faced in the medical field. Fogging often known as close to ground computation allows a well organize and disciplined computation to be done very near to the user end.

\subsection{Fogging in Medical Center}

Taking into consideration a particular ward in the hospice with numerous rooms in it and with a high requirement of medical attention for each admitted on their regular bodily observation, where an inferior prognosis would lead to a worst situations. The tactical placement of the fogging

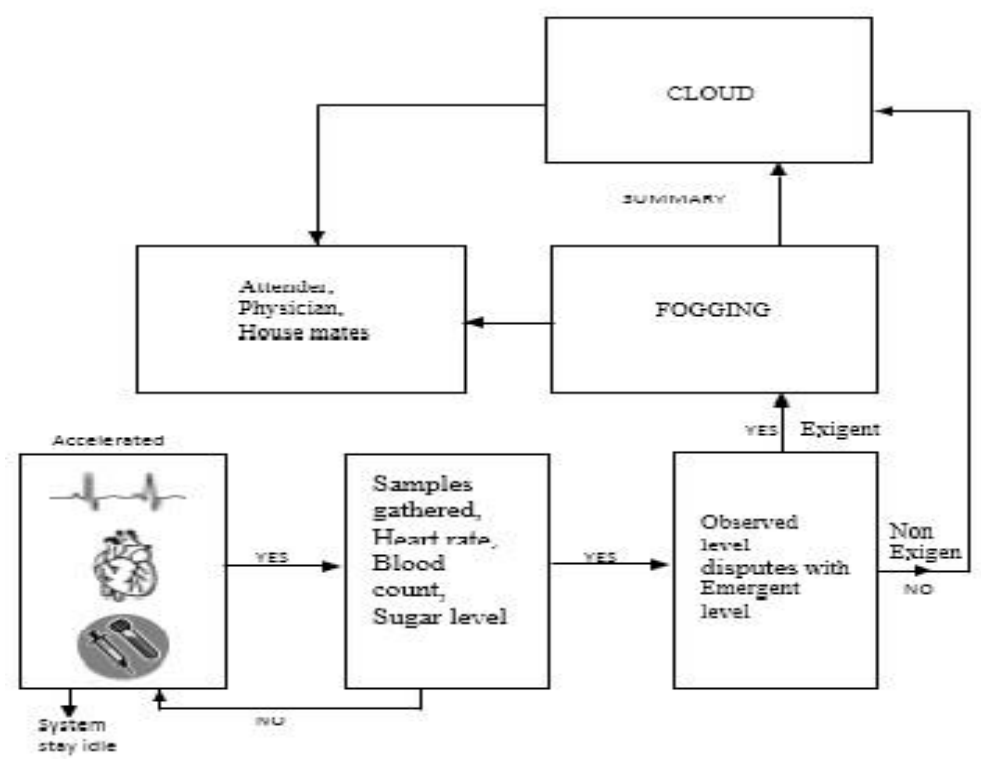

\section{Fig 2.Fogging in health center}

would appear insightful as it would update the medical professionals with their more critical and abnormal situations that require immediate actions to be taken, thus providing with a betterment in the quality of service. The fogging layer that is initiative of process to be done and controlled gathers all the particulars that being produced by the changes detected by the event detector and then operates on the particulars and moves it to the place or person it has 
J. Sustain. Wireless Syst.

Vol.01/ No. 02

Pages: 116-125

http://irojournals.com/irosws/

DOI: $\underline{\text { https://doi.org/10.36548/jsws.2019.2.004 }}$

to be sent. This computation involves the reception of event changes, gathers it and transiently stores it for further operating. The transiently stored information is sieved to extricate the needed particulars eliminating the superfluous encroaching to elude the repeated particulars and then it is lossless compressed and coalesced with various necessary details from the same point to keep down the using up of bandwidth. And the perused further for either moving the particulars the intimated place or person. This improvises the time taken to respond as the complete computation is done close to the user end and eludes the overload that would cause a heightened usage of bandwidth.

The primary structure adapted for a fogging supported medical center is well organized to have a heightened power conservation and a lengthened life span of energy storage devices. The evolved structure equipped with the expertise suggestions, prescription and guidance at the place where the disabled, injured or the infected is. Fogging does the collecting of symptoms incurred due to the bodily changes and further reviews it on a control lane escalating the virtue by averting the worst situations.

The proposed structure as in Fig 2 is entailed with multiple decks constituting the event detectors or the accelerometers, a mover, computations handling devices, remote storage devices and the expertise or physicians and the inmates to be informed are available dispersed in different deck.

The health center which is taken care by fogging is executed on the rostrum of internetwork of things burgeon where each edge devices that are wirelessly connected are supported by the embedded computing that are supported by object oriented high level software's for computations the event changes are detected, and the particulars are brought together and compacted and secured determining its mathematical average and perused the results are moved further for necessary actions and the summary of this computation is sent to the cloud this is case of a time intolerant application or information. The fogging structure connected to the edge device and cloud wireless conducts the essential monitoring, profiling, authorizing, endorsing, resources supervision, processing, actuating, and streaming by the software installed frame work of digitally communicated wireless devices, providing an unswerving machine to machine communication and data process without burdening the cloud. So whenever the device perceives a deformity the physician or the expertise and the care takers of the affected are notified without a human intervention.

Considering the injured cases with sudden aberrance in the sane conditions that disputes with the expected conditions immediate intimations are send to the respected authorities causing the urgent respond to be taken for the disabled. The encapsulation of the process is later send to the cloud for the subsequent reference for the physician or the housemates.

\subsection{Virtues of Fogging}

The fogging offers high virtue in terms of indemnity, latency and power consumption, as it is close to the grounds that is it near to the devices requesting for assist. The usage of constrained application protocol, and efficacious 
J. Sustain. Wireless Syst.

Vol.01/ No. 02

Pages: $116-125$

http://irojournals.com/irosws/

DOI: $\underline{\text { https://doi.org/10.36548/jsws.2019.2.004 }}$

sampling and winnowing methods, and capsuling of the networks and computation enables curtailed energy consumption in the ecosystem of fogging.

The infallible IP transport layer protocol enables data transferring across multiple IPs entails the structure to be tolerant for fault and provides with high security. The optimal schedulable nature based on the least time to go and least laxity first used by fogging resources management determines the proper allocation of tasks to either the cloud or fog itself causing a less latency and heightened throughput.

Finally the ability of the fog environments to add and remove the devices frequently due to their mobility enables the fog to have an enriched scalability and flexibility.

The construction of a fogging can be easily done using the available tools and can be devised according to the users need enabling business agility. They are prone to be adaptable to any environments including under water, tracks of the railways, vehicle, and factory floors and so on. It is an exquisite associate of cloud and internetwork of things eluding all the sources of difficulty faced while the information's are handled and empowers benefits from a single person to mighty firms providing real time analysis and processing.

\section{Result Evaluation}

The evaluation of this fogging based internetwork of things is done based on its impacts on response time, energy consumption and reliability and its comparison with cloud based structure without fogging is perused on the Ifogsim platform under different values and the results are acquired. With total of two hundred nodes being involved, with initial power assignment of 100 joules and simulation time of 100 seconds in the allotted arena of $40 * 2500 \mathrm{~m}$.

(a) Response time (RT)

The total time taken to answer back to the requisitions for a service also known as a glide past time of an enquiry from a system to its enquiry response. It is an assessment of system functionality. Where successful computations ends with low response time.it is given as in equation

Response time $=($ computation time - arriving time $) /$ processing time

ISSN: $2582-3167$

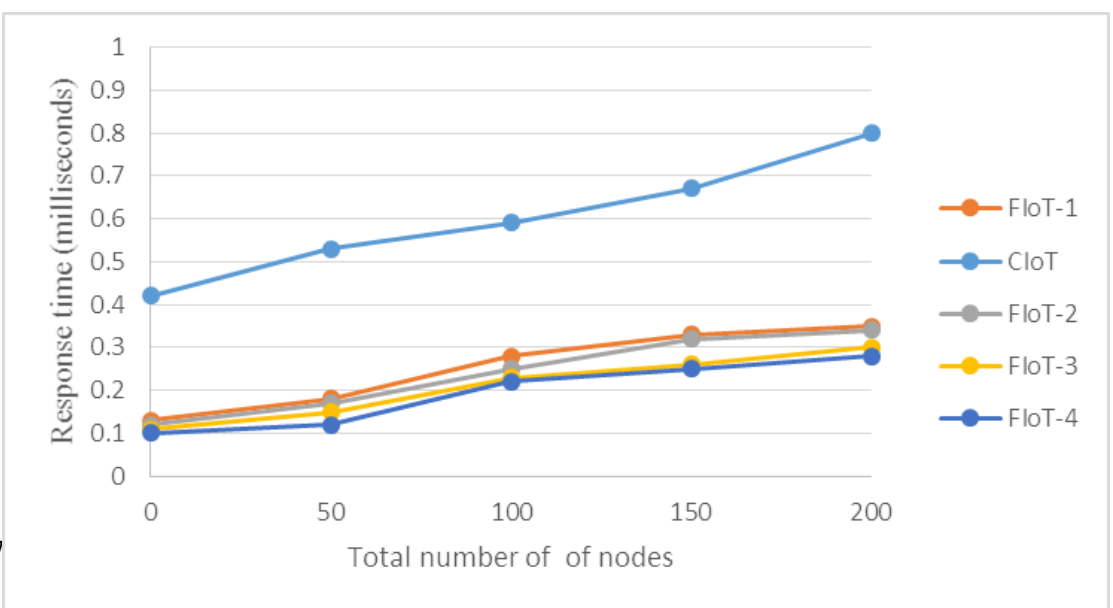


J. Sustain. Wireless Syst.

Vol.01/ No. 02

Pages: 116-125

http://irojournals.com/irosws/

DOI: https://doi.org/10.36548/jsws.2019.2.004

\section{Fig 3 Response time determination}

(2) the responses acquired from the graph in the Fig 3 gives the response time taken by the fog based internetwork of things are very less compared to the cloud based structure without fogging. Thus the reduced response time offers a quick decision making, diminished intrusions evading unexpected mishappenings.

\section{(b) Power consumption (Pc)}

Power consumption is given by successive summing of the transmitted power with the total number of devices used and summed up with the power spent on receiving data. The curve the Fig 4 shows the total power consumed by the interwork of things with fogging and without fogging .on executing with different sets of value fogging based structure shows nearly a forty percentage of reduced consumption compared to the other. Thus reporting to be economical in terms of power usage.

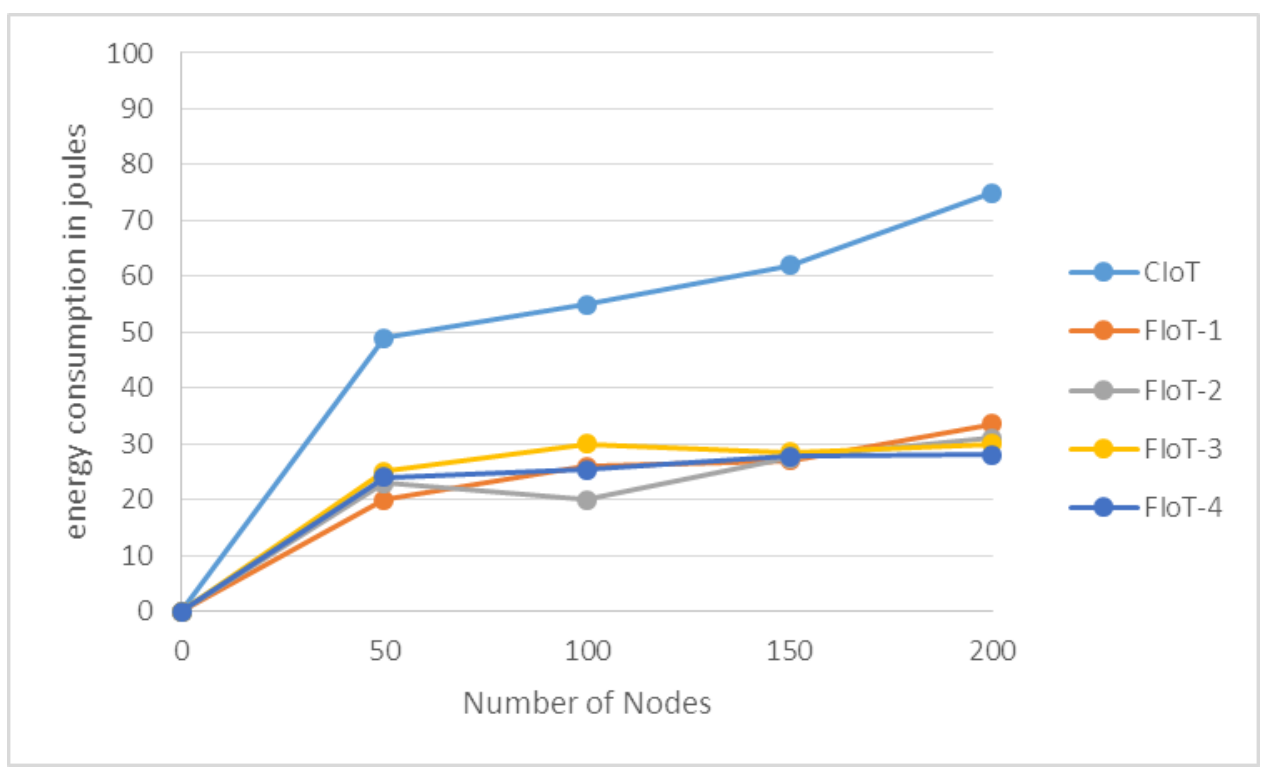

Fig 4.Power consumption determination

ISSN: 2582-3167 (online) 
J. Sustain. Wireless Syst.

Vol.01/ No. 02

Pages: 116-125

http://irojournals.com/irosws/

DOI: https://doi.org/10.36548/jsws.2019.2.004

The fogging allowing a close to user end operation ensures to be power saving as the entire computation is taken place near to the user end and only its encapsulation are sent to the cloud. The cut down in power consumption and the response time shows that major portion of the processing are done on the close to grounds so the number of data transmitted to the clouds would be lowered so the channel occupation towards the cloud also will be less as most of them are resolved closer to the grounds.

\section{Conclusion}

The expedient and luxurious life are the day to day dreams of many the connected networks of various information's and things offers that with the things brought together and systematized with the information's. This synchronization which overloaded with values created seems to be hard to be handled by the remote cloud structure so the proposed close to ground fogging structure offers a better information handling as they placed very close to the user end. It initiates as a merger of the cloud and edge devices to resolve the setbacks in the cloud structure and lends hand to internetwork of things reach a boundless prospective. Further this structure can be extended to other applications that are under exigent circumstance.

\section{References}

[1] Mutlag, Ammar Awad, Mohd Khanapi Abd Ghani, N. Arunkumar, Mazin Abed Mohamed, and Othman Mohd. "Enabling technologies for fog computing in healthcare IoT systems." Future Generation Computer Systems 90 (2019): 62-78.

[2] Elhoseny, Mohamed, K. Shankar, S. K. Lakshmanaprabu, Andino Maseleno, and N. Arunkumar. "Hybrid optimization with cryptography encryption for medical image security in internet of things." Neural Computing and Applications (2018): 1-15.

[3] Tran, Minh-Quang, Duy Tai Nguyen, Van An Le, Duc Hai Nguyen, and Tran Vu Pham. "Task Placement on Fog Computing Made Efficient for IoT Application Provision." Wireless Communications and Mobile Computing 2019 (2019).

[4] Muthanna, Ammar, Abdelhamied A. Ateya, Abdukodir Khakimov, Irina Gudkova, Abdelrahman Abuarqoub, Konstantin Samouylov, and Andrey Koucheryavy. "Secure IoT Network Structure Based on Distributed Fog Computing, with SDN/Blockchain." (2019).

[5] Wu, Fan, Taiyang Wu, and Mehmet Yuce. "An Internet-of-Things (IoT) Network System for Connected Safety and Health Monitoring Applications." Sensors 19, no. 1 (2019): 21.

[6] Abdellatif, Alaa Awad, Ahmed Emam, Carla-Fabiana Chiasserini, Amr Mohamed, Ali Jaoua, and Rabab Ward. "Edge-based compression and classification for smart healthcare systems: Concept, implementation and evaluation." Expert Systems with Applications 117 (2019): 1-14. 
J. Sustain. Wireless Syst.

Vol.01/ No. 02

Pages: 116-125

http://irojournals.com/irosws/

DOI: $\underline{\text { https://doi.org/10.36548/jsws.2019.2.004 }}$

[7] Aloi, Gianluca, Giancarlo Fortino, Raffaele Gravina, Pasquale Pace, and Giuseppe Caliciuri. "Edge Computing-Enabled Body Area Networks." In 2018 32nd International Conference on Advanced Information Networking and Applications Workshops (WAINA), pp. 349-353. IEEE, 2018.

[8] Cerina, Luca, Sara Notargiacomo, Matteo GrecoLuca Paccanit, and Marco Domenico Santambrogio. "A fog-computing architecture for preventive healthcare and assisted living in smart ambients." In 2017 IEEE 3rd International Forum on Research and Technologies for Society and Industry (RTSI), pp. 1-6. IEEE, 2017.

[9] Nakhkash, Mohammad R., Tuan Nguyen Gia, Iman Azimi, Arman Anzanpour, Amir M. Rahmani, and Pasi Liljeberg. "Analysis of Performance and Energy Consumption of Wearable Devices and Mobile Gateways in IoT Applications." (2019).

[10] Fiandrino, Claudio, Nicholas Allio, Dzmitry Kliazovich, Paolo Giaccone, and Pascal Bouvry. "Profiling Performance of Application Partitioning for Wearable Devices in Mobile Cloud and Fog Computing." IEEE Access (2019).

[11] Roca, Damian, Josue V. Quiroga, Mateo Valero, and Mario Nemirovsky. "Fog function virtualization: A flexible solution for IoT applications." In 2017 Second International Conference on Fog and Mobile Edge Computing (FMEC), pp. 74-80. IEEE, 2017.

[12] Newaz, SH Shah, Gyu Myoung Lee, Mohammad Rakib Uddin, Alaelddin Fuad Yousif Mohammed, and Jun Kyun Choi. "Towards realizing the importance of placing fog computing facilities at the central office of a PON." In 2017 19th International Conference on Advanced Communication Technology (ICACT), pp. 152-157. IEEE, 2017.

[13] Mahmoud, Mukhtar ME, Joel JPC Rodrigues, Kashif Saleem, Jalal Al-Muhtadi, Neeraj Kumar, and Valery Korotaev. "Towards energy-aware fog-enabled cloud of things for healthcare." Computers \& Electrical Engineering 67 (2018): 58-69. 